\title{
Pequenos mamíferos não-voadores (Didelphimorphia, Rodentia) em dois fragmentos de mata de restinga de Rio Grande, Planície Costeira do Rio Grande do Sul
}

\author{
Fernando Marques Quintela ${ }^{1,4}$, Maurício Beux Santos $^{2}$, Alexandre Uarth Christoff ${ }^{3}$ \& Adriana Gava ${ }^{2}$ \\ ${ }^{1}$ Programa de Pós-graduação em Biologia de Ambientes Aquáticos Continentais, Instituto de Ciências \\ Biológicas, Universidade Federal do Rio Grande - FURG, Av. Itália, Km 8, \\ CEP 96201-900, Rio Grande, RS, Brasil \\ ${ }^{2}$ Laboratório de Genética, Instituto de Ciências Biológicas, Universidade Federal do Rio Grande - FURG, \\ Av. Itália, Km 8, CEP 96201-900, Rio Grande, RS, Brasil \\ ${ }^{3}$ Departamento do Biologia, Museu de Ciências Naturais, Universidade Luterana do Brasil - ULBRA, \\ Av. Farroupilha, 8001, CEP 92420-280, Canoas, RS, Brasil \\ ${ }^{4}$ Autor para correspondência: Fernando Marques Quintela, e-mail: fmquintela@yahoo.com.br.
}

QUINTELA, F.M., SANTOS, M.B., CHRISTOFF, A.U. \& GAVA, A. Non-volant small mammals ((Didelphimorphia, Rodentia) in two forest fragments in Rio Grande, Rio Grande do Sul Coastal Plain, Brazil. Biota Neotrop. 12(1): http://www.biotaneotropica.org.br/v12n1/en/abstract?inventory+bn02412012012

\begin{abstract}
The restinga forests represent original vegetal formations in Coastal Plain of Rio Grande do Sul state. This work aimed to evaluate the species composition of non-volant small mammals in two restinga forests (peat forest and sandy riparian forest) in Rio Grande, Southern Rio Grande do Sul Coastal Plain. A total of 234 individuals belonging to three species of marsupials (Didelphidae: Cryptonanus guahybae, Didelphis albiventris, Lutreolina crassicaudata) and eight species of rodents (Cricetidae: Deltamys kempi, Holochilus brasiliensis, Oligoryzomys flavescens, O. nigripes, Oxymycterus nasutus, Scapteromys tumidus; Muridae: Mus musculus, Rattus rattus) was captured. The species C. guahybae, D. albiventris, D. kempi, $H$. brasiliensis, O. nigripes, $S$. tumidus and M. musculus were recorded in the peat forest while C. guahybae, D. albiventris, Lutreolina crassicaudata, D. kempi, O. flavescens, O. nigripes, S. tumidus and R. rattus occurred in the riparian sandy forest. Oligoryzomys nigripes and $S$. tumidus were the most abundant species in the peat forest, representing 40.4 and $22.1 \%$ of the total of captured individuals, respectively. The most abundant species in the riparian sandy forest were $O$. nigripes e D. albiventris, representing 63.4 and $12.4 \%$ of the total of captured individuals. Individuals of $C$. guahybae and $O$. nigripes were captured on trees (heights between 0.50 and $1.65 \mathrm{~m}$ ) while all individuals of the remaining species were captured on the ground.

Keywords: marsupials, rodents, peat forest, sandy riparian forest, Oligoryzomys nigripes, restinga.
\end{abstract}

QUINTELA, F.M., SANTOS, M.B., CHRISTOFF, A.U. \& GAVA, A. Pequenos mamíferos não-voadores (Didelphimorphia, Rodentia) em dois fragmentos de mata de restinga de Rio Grande, Planície Costeira do Rio Grande do Sul. Biota Neotrop. 12(1): http://www.biotaneotropica.org.br/v12n1/pt/abstract?inventory+ bn02412012012

Resumo: As matas de restinga representam formações vegetais originais na Planície Costeira do estado do Rio Grande do Sul. O objetivo deste trabalho foi avaliar a composição de espécies de pequenos mamíferos não-voadores em dois fragmentos de matas de restinga (mata palustre e mata arenosa ciliar) no município de Rio Grande, região sul da Planície Costeira do Rio Grande do Sul. Um total de 234 indivíduos pertencentes a três espécies de marsupiais (Didelphidae: Cryptonanus guahybae, Didelphis albiventris, Lutreolina crassicaudata) e oito espécies de roedores (Cricetidae: Deltamys kempi, Holochilus brasiliensis, Oligoryzomys flavescens, O. nigripes, Oxymycterus nasutus, Scapteromys tumidus; Muridae: Mus musculus, Rattus rattus) foi capturado. As espécies C. guahybae, D. albiventris, D. kempi, H. brasiliensis, O. nigripes, S. tumidus e M. musculus foram registradas no fragmento de mata palustre, enquanto que C. guahybae, D. albiventris, Lutreolina crassicaudata, $D$. kempi, O. flavescens, O. nigripes, S. tumidus e R. rattus ocorreram no fragmento de mata arenosa ciliar. Oligoryzomys nigripes e $S$. tumidus foram as espécies mais abundantes no fragmento de mata palustre, representando respectivamente 40,4 e $22,1 \%$ do total de indivíduos capturados. No fragmento de mata arenosa ciliar, as espécies mais abundantes foram $O$. nigripes e $D$. albiventris, representando respectivamente 63,4 e $12,4 \%$ do total de indivíduos capturados. Indivíduos de $C$. guahybae e $O$. nigripes foram capturados em estrato arbóreo (alturas entre 0,50 e 1,65 m) enquanto que todos os indivíduos das demais espécies foram capturados no solo. Palavras-chave: marsupiais, roedores, mata paludosa, mata arenosa ciliar, Oligoryzomys nigripes, restinga. 


\section{Introdução}

As formações de restinga compreendem as comunidades animais e vegetais das regiões costeiras arenosas e seus elementos físicos (Waechter 1985). Na região Sul do Brasil, estes ecossistemas típicos estão distribuídos desde o sul do Estado de Santa Catarina até o extremo sul do Estado do Rio Grande do Sul (Waechter 1985, Araújo 1992, Scherer et al. 2005), sendo que a maior parte dessa área é formada por bacias sedimentares quaternárias, com alguns afloramentos rochosos do Pré-Cambriano ao Jurásico (Waechter 1985). No Estado do Rio Grande do Sul, duas restingas estão presentes (São José do Norte e Rio Grande), dando forma a unidade fisiográfica da Planície Costeira Externa (Vieira 1984).

As matas de restinga representam comunidades vegetais originais na Planície Costeira do Rio Grande do Sul, compreendendo formações típicas tais como as matas palustres e matas arenosas ciliares. As matas palustres, também conhecidas como matas brejosas, turfosas, paludosas ou higrófilas (Waechter 1990), caracterizam-se por apresentar corpos d'água permanentemente ou periodicamente alagados por precipitação pluviométrica, sem margem bem definida e com fundo coberto por vegetação e lodo orgânico (Villwock et al. 1980). As mata ciliares, por sua vez, são formações silváticas associadas à margem de rios e outros cursos d'água, apresentando larguras variáveis (Marchiori 2004).

Estudos sobre comunidades de pequenos mamíferos não-voadores em formações florestais originais do Rio Grande do Sul são escassos, sendo conduzidos em fisionomias como Floresta de Araucária (Floresta Ombrófila Mista) (Cademartori et al. 2002, 2004, Dalmagro \& Vieira 2005, Iob \& Vieira 2008), ecótono Campo-Floresta de Araucária (Pedó 2005), Floresta Atlântica sensu strictu (Floresta Ombrófila Densa) (Horn 2005) e matas de restinga (Oliveira 1985, Langone 2007). Paralelamente a escassez de informações, observa-se a gradual perda e descaracterização dos ecossistemas florestais no Estado, uma vez que menos de 5\% da cobertura original de Mata Atlântica permanecem como fragmentos florestais, enquanto que pouco é conhecido sobre os remanescentes de mata de restinga no Bioma Pampa (Conservation International do Brasil et al. 2000). Em relação às matas de restinga, os processos de restabelecimento da cobertura vegetação original após distúrbios são ainda dificultados devido a características abióticas extremas (escassez de nutrientes e água, excesso de luz, mobilidade das dunas) e a diversificação das comunidades vegetais (Scherer et al. 2007). Frente a isso, o presente trabalho apresenta a composição e riqueza de espécies de pequenos mamíferos não-voadores (Didelphimorphia, Rodentia) em dois fragmentos de mata de restinga (mata palustre e mata arenosa ciliar) na região sul da Planície Costeira do Estado do Rio Grande do Sul.

\section{Material e Métodos}

\section{1. Área de estudo}

Este estudo foi conduzido em dois fragmentos de mata de restinga localizados no município de Rio Grande, Planície Costeira do Estado do Rio Grande do Sul. O clima no município é classificado como mesotérmico superúmido (Cfa de Köppen) com estações bem definidas e temperaturas variando de $9,5^{\circ} \mathrm{C}$ no mês de Julho (média das mínimas) a $27,2^{\circ} \mathrm{C}$ no mês de Janeiro (média das máximas). A precipitação média mensal é de $104 \mathrm{~mm}$ e os meses mais chuvosos são julho, agosto e setembro. As cotas altimétricas variam do nível do mar a cerca de sete metros (Vieira 1983). O município está inserido no bioma Pampa (Instituto... 2004).

$\mathrm{O}$ fragmento de mata palustre amostrado é localmente conhecido como "Mata da Estrada Velha" (32 07' S e 52 09' O), cobre uma área aproximada de $220.000 \mathrm{~m}^{2}$ e está inserido na Área de Preservação Permanente $n^{\circ} 6$ (APP6) (Figura 1). Neste fragmento foram amostradas formações com alta densidade de indivíduos arbóreos e uma formação do tipo clareira. Nas primeiras formações a vegetação é composta principalmente por espécies arbóreas (Erytrina crista-galli, Syagrus romanzoffiana, Ficus cestrifolia) e macrófitas aquáticas tais como Ranunculus apiifolius, Hydrocotyle ranunculoides, Centella asiatica, Enydra sp., Scirpus spp., Saggitaria montevidensis, Senecio bonariensis, Bromelia anthiacantha, Eryngium pandalifolium, entre outras. A vegetação na clareira é composta principalmente por gramíneas, herbáceas (E. pandanifolium, Polygonum hydropiperoides, Hydrocotyle ranunculoides, Enydra sp.) e arbóreas esparsas (E. crista-galli). Um período de alagamento de secções do fragmento ocorreu nos meses de maio a novembro, sendo a maior profundidade registrada de $45 \mathrm{~cm}$.

$\mathrm{O}$ fragmento de mata arenosa ciliar amostrado localiza-se às margens do Arroio Bolaxa ( $32^{\circ} 09^{\prime} \mathrm{S}$ e $\left.52^{\circ} 11^{\prime} \mathrm{O}\right)$ e está inserido na Área de Proteção Ambiental da Lagoa Verde (Figura 1). Foi amostrado um trecho no interior e borda da mata localizado na margem norte do arroio, sendo a vegetação nessa área composta principalmente por espécies arbóreas e herbáceas tais com Ficus organensis, F. luschnatiana, Erythrina crista-galli, Myrsine parvifolia, Schinus terenbinthifolius, Salix humbodtiana, Bromelia anthiacantha, Eryngium elegans, E. pandanifolium, Schoenoplectus californicus, Scirpus giganteus, Cyperus spp. Algumas seções da mata adjacentes à várzea do arroio Bolaxa permaneceram alagadas nos meses de julho a outubro, sendo a maior profundidade registrada de $83 \mathrm{~cm}$. A distância entre os fragmentos é de aproximadamente $5 \mathrm{~km}$.

\section{Amostragem biológica}

As amostragens foram realizadas sazonalmente em sessões de captura com duração de 14 noites consecutivas em cada fragmento. Noventa armadilhas de arame de quatro diferentes tamanhos $(78 \times 32 \times 36 \mathrm{~cm} ; 43 \times 21 \times 20 \mathrm{~cm} ; 32 \times 15 \times 15 \mathrm{~cm} ; 30 \times 11 \times 10 \mathrm{~cm})$ foram instaladas ao longo de um transecto linear de $900 \mathrm{~m}$ de extensão, distantes dez metros entre si. Um total de 30 armadilhas foi instalado no sub-bosque, a alturas entre 0,50 e 3,5 m. As armadilhas foram arranjadas em uma sequiência repetida de duas armadilhas no solo e uma em sub-bosque. Foi utilizado com isca óleo de fígado de bacalhau e amendoim. Em cada fragmento foi instalada uma linha de armadilhas de queda (pitfall traps) constituídas por seis baldes de $52 \mathrm{~L}$ distantes $5 \mathrm{~m}$ entre si, conectados por uma tela plástica de $50 \mathrm{~cm}$ de altura e $30 \mathrm{~m}$ de extensão. As armadilhas de queda permaneceram abertas durante toda a sessão de capturas, sendo verificadas diariamente. $\mathrm{O}$ esforço de captura por amostragem sazonal foi de 2.520 armadilhas-noite e 168 baldes-noite (1.260 armadilhas-noite e 84 baldes-noite em cada fragmento), sendo o esforço de captura total 10.080 armadilhas-noite e 672 baldes-noite (5.040 armadilhas-noite e 336 baldes-noite em cada fragmento).

Exceto por Didelphis albiventris Lund, 1840, todos os indivíduos capturados foram coletados para preparação de pele e crânio e extração de material medular femural para posterior análise citogenética. Três indivíduos de $D$. albiventris foram coletados enquanto que os demais foram marcados com brincos metálicos numerados e posteriormente soltos. Roedores foram identificados com base nos dados cariotípicos obtidos, sendo observados o número diplóide (2n) e o número de braços autossômicos (NA). Em relação às capturas no sub-bosque, foi medida a altura (em metros) do solo a base da armadilha. Crânios e peles dos indivíduos foram depositados na coleção mastozoológica do Museu de Ciências Naturais da Universidade Luterana do Brasil (MCNU). 
Pequenos mamíferos em matas de restinga

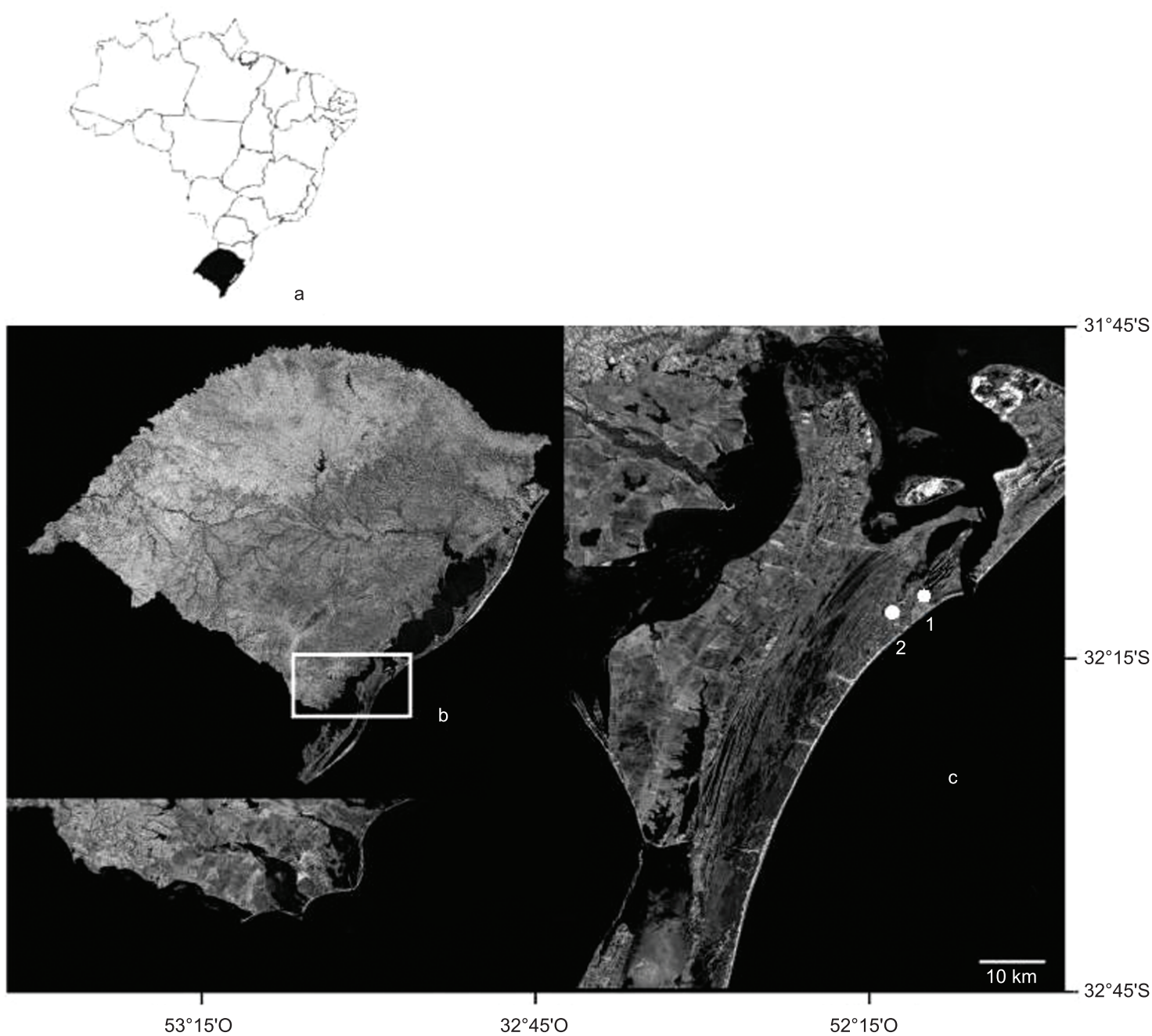

Figura 1. Localização do Estado do Rio Grande do Sul (a), parte da região sul da Planície Costeira (b) e área de estudo (c), sendo: 1) fragmento de mata palustre, 2) fragmento de mata arenosa ciliar.

Figure 1. Location of Rio Grande do Sul State (a), southern Coastal Plain (b) and study area (c), being: 1) peat forest, 2) sandy riparian forest.
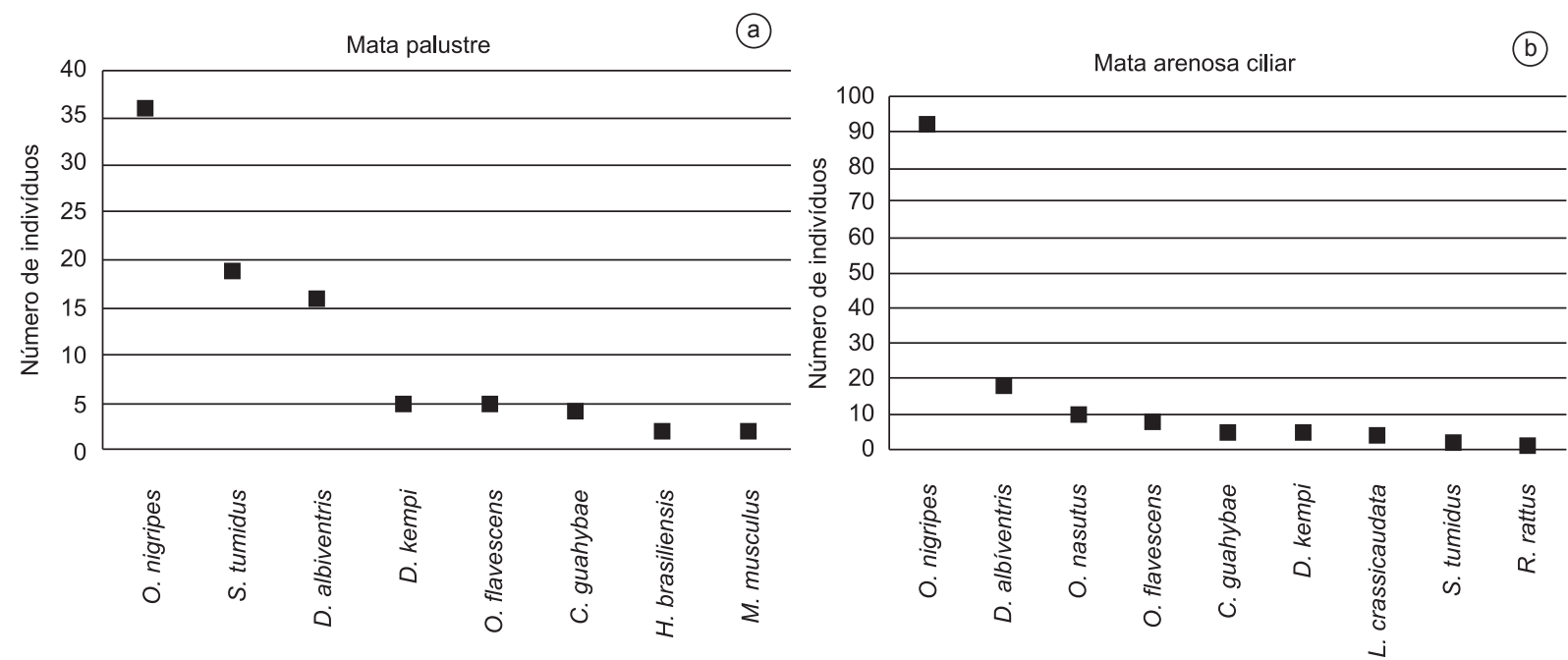

Figura 2. Rank de espécies de pequenos mamíferos não-voadores registrados em um fragmento de mata palustre (a) e um fragmento de mata arenosa ciliar (b) no município de Rio Grande, Estado Rio Grande do Sul, Brazil.

Figure 2. Rank of species of non-volant small mammals recorded in a peat forest fragment (a) and a sandy riparian forest (b) in Rio Grande County, Rio Grande do Sul State, Brazil. 
Tabela 1. Número de indivíduos capturados e porcentagem relativa de capturas (entre parênteses) das espécies de pequenos mamíferos não-voadores em amostragens sazonais realizadas em um fragmento de mata palustre (MP) e um fragmento de mata arenosa ciliar (MC) no município de Rio Grande, Estado Rio Grande do Sul, Brazil.

Table 1. Number of captured individuals and relative percentage of captures (between parentheses) of non-volant small mammals on seasonal sampling performed in a peat forest fragment (MP) and a sandy riparian forest (MC) in Rio Grande County, Rio Grande do Sul State, Brazil.

\begin{tabular}{|c|c|c|c|c|c|c|c|c|}
\hline \multirow{2}{*}{ Taxon } & \multicolumn{2}{|c|}{ Verão } & \multicolumn{2}{|c|}{ Outono } & \multicolumn{2}{|c|}{ Inverno } & \multicolumn{2}{|c|}{ Primavera } \\
\hline & MP & $\mathrm{MC}$ & MP & $\mathrm{MC}$ & MP & $\mathrm{MC}$ & MP & $\mathrm{MC}$ \\
\hline \multicolumn{9}{|l|}{ Didelphimorphia } \\
\hline \multicolumn{9}{|l|}{ Didelphidae } \\
\hline $\begin{array}{l}\text { Cryptonanus guahybae } \\
\text { (Tate, 1931) }\end{array}$ & $1(25 \%)$ & & $2(50 \%)$ & $1(20 \%)$ & $1(25 \%)$ & $2(40 \%)$ & & $2(40 \%)$ \\
\hline $\begin{array}{l}\text { Didelphis albiventris } \\
\text { (Lund, 1840) }\end{array}$ & $4(25 \%)$ & $6(33.3 \%)$ & $9(56.2 \%)$ & $4(22.2 \%)$ & $1(6.3 \%)$ & & $2(12.5 \%)$ & $8(44.4 \%)$ \\
\hline $\begin{array}{l}\text { Lutreolina crassicaudata } \\
\text { (Desmarest, 1804) }\end{array}$ & & $4(100 \%)$ & & & & & & \\
\hline \multicolumn{9}{|l|}{ Rodentia } \\
\hline \multicolumn{9}{|l|}{ Cricetidae } \\
\hline Deltamys kempi (Thomas, 1817) & $1(10 \%)$ & $5(50 \%)$ & $3(30 \%)$ & & $1(10 \%)$ & & & \\
\hline $\begin{array}{l}\text { Holochilus brasiliensis } \\
\text { (Desmarest, 1819) }\end{array}$ & $2(100 \%)$ & & & & & & & \\
\hline $\begin{array}{l}\text { Oligoryzomys flavescens } \\
\text { (Waterhouse, 1837) }\end{array}$ & & $5(62.5 \%)$ & $3(60 \%)$ & & $2(40 \%)$ & $2(25 \%)$ & & $1(12.5 \%)$ \\
\hline $\begin{array}{l}\text { Oligoryzomys nigripes } \\
\text { (Olfers, 1818) }\end{array}$ & & $5(5.4 \%)$ & $9(25 \%)$ & $35(38 \%)$ & $26(72.2 \%)$ & $41(44.6 \%)$ & $1(2.8 \%)$ & $11(11.9 \%)$ \\
\hline $\begin{array}{l}\text { Oxymycterus nasutus } \\
\text { (Waterhouse, } 1837 \text { ) }\end{array}$ & & $2(20 \%)$ & & $3(30 \%)$ & & & & $5(50 \%)$ \\
\hline $\begin{array}{l}\text { Scapteromys tumidus } \\
\text { (Waterhouse, 1837) }\end{array}$ & & $2(9 \%)$ & $6(29 \%)$ & & $5(24 \%)$ & & $8(38 \%)$ & \\
\hline \multicolumn{9}{|l|}{ Muridae } \\
\hline Mus musculus (Linnaeus, 1758) & $2(100 \%)$ & & & & & & & \\
\hline Rattus rattus (Linnaeus, 1758) & & $1(100 \%)$ & & & & & & \\
\hline Total & $10(11.2 \%)$ & $30(20.7 \%)$ & $32(35.9 \%)$ & $43(29.6)$ & $36(40.4 \%)$ & $45(31 \%)$ & $11(12.3 \%)$ & $27(18.6 \%)$ \\
\hline
\end{tabular}

\section{Resultados}

Foram capturados 234 indivíduos pertencentes a três espécies de marsupiais e oito espécies de roedores (Tabela 1). Destes, 89 indivíduos foram capturados no fragmento de mata palustre enquanto que 145 foram capturados no fragmento de arenosa ciliar. Ocorreram 13 recapturas de Didelphis albiventris na mata palustre e 12 na mata arenosa ciliar. O sucesso de captura total foi $3 \%$ para as armadilhas Tomahawk e 7,1\% para as armadilhas-de-queda. Indivíduos de todas as espécies foram capturados em armadilhas Tomahawk enquanto que indivíduos de Cryptonanus guahybae (Tate, 1931), Deltamys kempi (Thomas, 1817) $(2 \mathrm{n}=38$; NA = 38), Holochilus brasiliensis (Desmarest, 1819) $(2 \mathrm{n}=42$; NA $=64)$, Oligoryzomys flavescens (Waterhouse, 1837) $(2 \mathrm{n}=64-67$; $\mathrm{NA}=66-69)$, O. nigripes (Olfers, 1818) $(2 \mathrm{n}=62 ; \mathrm{NA}=81-82)$, Oxymycterus nasutus Waterhouse, $1837(2 \mathrm{n}=54 ; \mathrm{NA}=64) \mathrm{e}$ Scapteromys tumidus (Waterhouse, 1837) $(2 \mathrm{n}=24$; NA $=40)$ foram também amostrados em armadilhas de queda.

Oligoryzomys nigripes e S. tumidus foram as espécies mais abundantes no fragmento de mata palustre, representando respectivamente 40,44 e 21,34\% do total de indivíduos capturados. No fragmento de mata arenosa ciliar as espécies mais abundantes foram $O$. nigripes e $D$. albiventris, representando respectivamente 63,44 e 12,41\% do total de capturas. A abundância relativa das demais espécies é mostrada na Figura 1.
Indivíduos de C. guahybae, O. flavescens e $O$. nigripes foram capturado no sub-bosque, em alturas variando entre 0,50 e 1,65 m. Considerando os estratos de captura, o sucesso de captura foi $2,76 \%$ para solo e $0,53 \%$ para sub-bosque na mata palustre e $4,07 \%$ para solo e $1,19 \%$ para sub-bosque na mata arenosa ciliar.

\section{Discussão}

As assembléias de pequenos mamíferos dos fragmentos de mata de restinga amostrados no presente estudo apresentaram riquezas de espécies semelhantes a aquelas encontradas em estudos realizados em outras formações florestais do Brasil meridional, apesar das diferenças em relação à composição e dominância de espécies. Cherem \& Perez (1996) registraram a ocorrência de três marsupiais e oito roedores no norte de Santa Catarina, sendo Akodon montensis (Thomas, 1913) a espécie mais capturada em Floresta de Araucária e $O$. nigripes em Mata Ciliar. Em uma área de Floresta Ombrófila Densa Submontana na Ilha de Santa Catarina, Graipel et al. (2006) amostraram quatro marsupiais e sete roedores, com dominância de Euryoryzomys russatus (Wagner, 1848). Dalmagro \& Vieira (2005) registraram um marsupial e seis roedores em Floresta de Araucária no Parque Nacional de Aparados da Serra, Rio Grande do Sul, sendo O. nigripes a espécie mais presente. Horn (2005) amostrou três marsupiais e oito roedores em uma mata palustre localizada no extremo norte da Planície Costeira do Rio Grande do Sul (bioma 
Mata Atlântica), sendo o A. montensis a espécie mais abundante. Em um fragmento de mata de restinga na região sul da Planície Costeira Interna do Rio Grande do Sul, Langone (2007) registrou duas espécie de marsupiais e oito roedores, com dominância de $O$. nigripes. Considerando-se os trabalhos supramencionados e os dados obtidos no presente estudo, observa-se que $A$. montensis e $O$. nigripes representam, portanto, os pequenos mamíferos mais abundantes na maioria dos estudos conduzidos em formações florestais do Brasil meridional.

As diferenças observadas em relação à composição de espécies nas distintas assembléias dos podem estar relacionadas a dessemelhanças estruturais entre os habitats. Em sistemas florestais, variáveis como o grau de fragmentação, complexidade vegetal e diversidade de habitats e microhabitats refletem em diferenças quanto os padrões de composição e abundância de espécies de pequenos mamíferos (Pardini 2004, Pardini et al. 2005, Pardini \& Umetsu 2006). Nesse contexto, é importante ressaltar a ausência de Akodon reigi González, Langguth \& Oliveira, 1998 no presente estudo, espécie bem representativa nas matas de restinga amostradas por Oliveira (1985) e Langone (2007), distantes respectivamente cerca de 55 e $40 \mathrm{~km}$ dos fragmentos de Rio Grande. González \& Lanfranco (2010), no entanto, citam a ocorrência de $A$. reigi em matas ciliares e ressaltam que a espécie pode se encontram em baixas densidades, além de sofrer importantes flutuações populacionais. Outra espécie do gênero Akodon, A. azarae, foi também amostrada por Oliveira (1985) em interior e borda de mata psamófila. Akodon azarae é considerado uma espécie habitat generalista, com ocorrência registrada também em formações ciliares (González \& Lanfranco 2010).

Exceto por D. kempi e H. brasiliensis, todas as demais espécies autóctones são citadas como ocorrentes em sistemas florestais (Achaval et al. 2004, Bonvicino et al. 2008, González \& Lanfranco 2010). Deltamys kempi é citado com ocorrente em ambientes pantanosos, tais como bordas de banhados e campos alagadiços, geralmente sem árvores (González \& Pardiñas 2002, Bonvicino et al. 2008). Holochilus brasiliensis habita principalmente em juncais e pradarias em banhados, margens de lagoas e arroios e rios (González \& Lanfranco 2010). A presença destas duas espécies na área de estudo, portanto, indica uma influência dos sistemas alagados de áreas abertas sobre os fragmentos florestais. Também deve ser considerado o caráter do estrato herbáceo bem desenvolvido em ambos os fragmentos, representado principalmente por apiáceas na mata palustre e bromeliáceas e asteráceas na mata arenosa ciliar, o que pode vir a favorecer a dispersão destas espécies.

Cryptonanus guahybae é uma espécie considerada com Dados Deficientes na Lista Vermelha das Espécies Ameaçadas da IUCN (Costa et al. 2008) e sua distribuição é restrita à Planície Costeira do Rio Grande do Sul (Voss et al. 2005, Rossi \& Bianconi 2011). A baixa representatividade numérica deste didelfídeo no presente estudo pode indicar que se trate de uma espécie naturalmente rara, ocorrendo em baixas densidades. Uma vez que há uma carência de dados para a avaliação de seu status de conservação, torna-se necessário assegurar a proteção das populações já localizadas, mantendo a integridade de seus habitats.

Apesar das pequenas proporções, as matas de restinga amostradas no presente estudo sustentam uma representativa fauna de pequenos mamíferos. Considerando-se que inventários representam o ponto de partida para demais estudos em comunidades biológicas, muitos aspectos biológicos e ecológicos das espécies amostradas precisam ainda ser investigados. Densidades, flutuações populacionais temporais, áreas de vida, padrões reprodutivos e outros parâmetros podem futuramente ser elucidados com a aplicação dos métodos apropriados.

\section{Agradecimentos}

Somos gratos a Chyntia Ibarra e Mauro C. L. M. Oliveira pelo auxílio em campo, Eduardo L. Coelho, Susi M. Pacheco, Eduardo R. Secchi and Elton P. Colares pelo apoio logístico, a Coordenação de Aperfeiçoamento de Pessoal do Nível Superior (CAPES) pela bolsa concedida ao primeiro autor e ao Instituto Chico Mendes pela licença de coleta (processo $\mathrm{n}^{\mathrm{o}} 325-1$ ).

\section{Referências Bibliográficas}

ACHAVAL, F., CLARA, M. \& OLMOS, A. 2004. Mamíferos de la República Oriental Del Uruguay. Imprimex, Montevideo.

ARAÚJO, D.S.D. 1992. Vegetation types of Sandy coastal plains of tropical Brazil: a first approximation. In Coastal Plant Communities of Latin America (U. Seeliger, ed.). Academy Press, New York, p.337-347.

BONVICINO, C.R., OLIVEIRA, J.A. \& D'ANDREA, P.S. 2008. Guia dos roedores do Brasil, com chaves para gêneros baseadas em caracteres externas. Centro Pan-Americano de Febre Aftosa - OPAS/OMS, Rio de Janeiro.

CADEMARTORI, C.V., FÁBIAN, M.E. \& MENEGHETI, J.O. 2004. Variações na abundância de roedores (Rodentia, Sigmodontinae) em duas áreas de floresta ombrófila mista, Rio Grande do Sul, Brasil. Rev. Bras. Zooc. 6(2):147-167.

CADEMARTORI, C.V., MARQUES, R.V., PACHECO, S.M., BAPTISTA, L.R.M. \& GARCIA, M. 2002. Roedores ocorrentes em Floresta Ombrófila Mista (São Francisco de Paula, Rio Grande do Sul) e a caracterização de seu hábitat. Comum. Mus. Ciênc. Tecnol. PUCRS, Sér. Zool. 15(1):61-86.

CHEREM, J.J. \& PEREZ, D.M. 1996. Mamíferos terrestres de floresta de araucária no município de Três Barras, Santa Catarina, Brazil. Biotemas 9(2):29-46.

CONSERVATION INTERNACIONAL DO BRASIL, FUNDAÇÃO SOS MATA ATLÂNTICA, FUNDAÇÃO BIODIVERSITAS, INSTITUTO DE PESQUISAS ECOLÓGICAS, SÃO PAULO. Secretaria do Meio Ambiente do Estado de São Paulo - SEMAD \& Instituto Estadual de Florestas. 2000. Avaliação e ações prioritárias para a conservação da biodiversidade da Mata Atlântica e Campos Sulinos. Ministério do Meio Ambiente, Brasília.

COSTA, L., ASTUA DE MORAES, D., BRITO, D., SORIANO, P., LEW, D. \& DELGADO, C. 2008. Cryptonanus guahybae. In: IUCN Red listo $\mathrm{f}$ Threatened Species (International Union for Conservation of Nature). version 2011.2 http://www.iucnredlist.org/apps/redlist/details/136705/0 (ultimo acesso em 13/11/2011).

DALMAGRO, A.D. \& VIEIRA, E.M. 2005. Patterns of habitat utilization of small rodents in an area of Araucaria forest in Southern Brazil. Austral Ecol. 30:353-362. http://dx.doi.org/10.1111/j.1442-9993.2005.01447.x

GONZÁLEZ, E.M., LANFRANCO, J.A.M. 2010. Mamíferos de Uruguay. Guía de campo e introducción a su estúdio y conservación. Banda Oriental, Museo Nacional de Historia Natural, Vida Silvestre Uruguay, Montevideo.

GONZÁLEZ, E.M. \& PARDIÑAS, F.J. 2002. Deltamys kempi. Mammal. Spec. 771:1-4.

GRAIPEL, M.E., CHEREM, J.J., MONTEIRO-FILHO, E.L.A. \& GLOCK, L. 2006. Dinâmica populacional de marsupiais e roedores no Parque Municipal da Lagoa do Peri, Ilha de Santa Catarina, Sul do Brasil. Mastozool. Neotrop. 13(1):31-49.

HORN, G.B. 2005. A assembléia de pequenos mamíferos da floresta paludosa do Faxinal, Torres-RS: sua relação com a borda e o roedor Akodon motensis (Rodentia, Muridae) como potencial dispersos de sementes endozoocóricas. Dissertação de mestrado, Universidade Federal do Rio Grande do Sul, Porto Alegre.

INSTITUTO BRASILEIRO DE GEOGRAFIA EESTATÍSTICA - IBGE. 2004. Mapa de Biomas e de Vegetação. http://www.ibge.gov.br/home/ presidencia/noticias/noticia_visualiza.php?id_noticia $=169>$. (último acesso em: 10/11/2011. 
IOB, G. \& VIEIRA, E.M. 2008. Seed predation of Araucaria angustifolia (Araucariaceae) in the Brazilian Araucaria Forest: influence of deposition site and comparative role of small and 'large' mammals. Plant Ecol. 198:185-196.

LANGONE, P.Q. 2007. Importância da matriz e das características do habitat sobre a assembléia de pequenos mamíferos em fragmentos de restinga no sul do Brasil. Dissertação de mestrado, Universidade Federal do Rio Grande, Porto Alegre.

MARCHIORI, J.N.C. 2004. Fitogeografia do Rio Grande do Sul: campos sulinos. EST, Porto Alegre.

OLIVEIRA, L.F.B. 1985. Estrutura e ordenação espaço-temporal de uma congregação de roedores no sul da Planície Costeira do Rio Grande do Sul, Brasil (Mammalia, Rodentia, Cricetidae). Dissertação de mestrado, Universidade Federal do Rio Grande do Sul, Porto Alegre.

PARDINI, R. 2004. Effects of Forest fragmentation on small mammals in na Atlantic Forest landscape. Biodivers. Conserv. 13:2467-2586.

PARDINI, R., SOUZA, S.M., BRAGA-NETO, R. \& METZGER, J.P. 2005. The role of Forest structure, fragment size and corridors in maintaining small mammals abundance diversity in an Atlantic Forest landscape. Biol. Conserv. 124:253-266. http://dx.doi.org/10.1016/j.biocon.2005.01.033

PARDINI, R. \& UMETSU, F. 2006. Pequenos mamíferos não-voadores da Reserva Florestal do Morro Grande - distribuição das espécies e da diversidade em uma área de Mata Atlântica. Biota Neotrop. 6(2): http:// www.biotaneotropica.org.br/v6n2/pt/abstract?article+bn006060022006

PEDÓ, E. 2005. Assembléia de pequenos mamíferos não-voadores em área de ecótono campo - floresta com Araucária na região dos Campos de Cima da Serra, Rio Grande do Sul. Dissertação de mestrado, Universidade Federal do Rio Grande do Sul, Porto Alegre.
ROSSI, R.V. \& BIANCONI, G.V. 2011. Ordem Didelphiomorpha. In Mamíferos do Brasil (N.R. Reis, A.L. Peracchi, W.A., Pedro \& I.P. Lima, eds.). Nelio R. dos Reis, Londrina, p.31-70.

SCHERER, A., MARASCHIN-SILVA, F. \& BAPTISTA, L.R.M. 2005. Florística e estrutura do componente arbóreo de matas de Restinga arenosa no Parque Estadual de Itapuã, RS, Brasil. Acta Bot.Bras. 19(4):717-726.

SCHERER, A., MARASCHIN-SILVA, F. \& BAPTISTA, L.R.M. 2007. Regeneração arbórea num capão de restinga no Rio Grande do Sul, Brasil. Iheringia, Sér. Bot. 62(1-2):89-98.

VIEIRA, E.F. 1983. Rio Grande: geografia física, humana e econômica. Sagra, Porto Alegre.

VIEIRA, E.F. 1984. Rio Grande do Sul: geografia física e vegetação. Sagra, Porto Alegre.

VILLWOCK, J.A., DEHNHARDT, E.A., LOSS, E.L. \& HOFMEISTER, T. 1980. Turfas da província costeira do Rio Grande do Sul - Geologia depósito Águas claras. In Congresso Brasileiro de Geologia (Sociedade Brasileira de Geologia, coord.). Sociedade Brasileira de Geologia, Camboriú, p.500-512.

VOSS, R.S., LUNDE, D.P. \& JANSA, S.A. 2005. On the contents of Gracilinanus Gardner and Creighton, 1989, with the Description of a Previously Unrecognized Clade of Small Didelphid Marsupials. Am. Mus. Novit. 3482:1-34. http://dx.doi.org/10.1206/00030082(2005)482[0001:OTCOGG]2.0.CO;2

WAECHTER, J.L. 1985. Aspectos ecológicos da vegetação de restinga no Rio Grande do Sul, Brasil. Comum. Mus. Ciênc. Tecnol. PUCRS, Sér. Bot. 33:49-68.

WAECHTER, J.L. 1990. Comunidades vegetais das restingas do Rio Grande do Sul. In Simpósio de Ecossistemas da Costa Sul e Sudeste Brasileira (Academia de Ciências do Estado de São Paulo, org.). Academia de Ciências do Estado de São Paulo, Águas de Lindóia, v.3, p.228-248. 